\title{
Morphology of Polyethylene-Carbon Black Composites
}

\author{
G. BEAUCAGE, ${ }^{1}$ S. RANE, ${ }^{1}$ D. W. SCHAEFER, ${ }^{1}$ G. LONG, ${ }^{2}$ D. FISCHER ${ }^{2}$ \\ ${ }^{1}$ University of Cincinnati, Cincinnati, Ohio 45221-0012 \\ ${ }^{2}$ National Institute of Standards and Technology, Gaithersburg, Maryland 20885
}

Received 6 August 1998; revised 6 November 1998; accepted 9 November 1998

\begin{abstract}
Carbon black is a common polymer additive that is used for reinforcement and for its ability to enhance physical properties, such as conductivity. This article pertains to an X-ray scattering (SAXS) study of a conductive grade of carbon black and carbon black-polymer composites. The scattering pattern for such blacks displays a surface-fractal-like power-law decay over many decades in scattering vector $q$. It is often assumed that small-angle scattering from carbon black aggregates can be described in terms of surface-fractal models, related to particles with fractally rough surfaces. Such self-similar surface roughness is usually easy to identify by microscopy; however, electron microscopy from these blacks fails to support this assumption. It is proposed here that this apparent surface-fractal scattering actually represents a more complicated morphology, including overlapping structural features and a power-law scaling of polydispersity. One use of conductive black-polyethylene composites is in circuit protection devices where resistive heating leads to a reversible association of carbon black aggregates that controls switching between a conductive and a nonconductive state. Scattering can be used as an in situ tool to observe the morphological signature of this reversible structural change. Scattering patterns support a model for this switching based on local enhancement of concentration and the formation of linear agglomerates associated with the matrix polymer's semicrystalline morphology. () 1999 John Wiley \& Sons, Inc. J Polym Sci B: Polym Phys 37: 1105-1119, 1999

Keywords: small-angle scattering; polyethylene; poly(methyl methacrylate); carbon black; filler; composite; conductivity; percolation
\end{abstract}

\section{INTRODUCTION}

The morphology of composite materials often presents an enigma to materials scientists since the overlap of features on many length scales can present a formidable challenge to the interpretation of microstructural data. This article focuses on composites of semicrystalline polymers with carbon black. Because carbon black (CB) absorbs most visible light, even when loaded at less than a percent, techniques such as optical microscopy and light scattering are precluded. Further, the

Correspondence to: G. Beaucage

Journal of Polymer Science: Part B: Polymer Physics, Vol. 37, 1105-1119 (1999) (C) 1999 John Wiley \& Sons, Inc. CCC 0887-6266/99/111105-15 conductivity of carbon black, combined with the insulating properties of the polymer matrix, lead to difficulties in the use of electron microscopy. Even when electron microscopy is successful, the superposition of three-dimensional (3-D) structure in these fillers can lead to difficulty in the interpretation of micrographs. Small-angle X-ray scattering (SAXS) and small-angle neutron scattering (SANS) scattering are likely candidates for the study of these materials because they can be used to probe 3-D space. One problem with conventional pinhole or Kratky SAXS cameras is that the size scale probed rarely exceeds $200 \AA$. For the conductive black, of interest here, the reported mean primary particle size is $800 \AA$, and the closely packed aggregates of these primary parti- 
cles are of submicrometer size. Agglomerates of these aggregates may reach $10 \mathrm{~s}$ of micrometers in size. In this article, data from an ultra-smallangle X-ray camera (USAXS) are combined with data from a conventional pinhole camera to elucidate morphologies from about $1 \AA$ to about 1 $\mu \mathrm{m}$. The analysis of data that contain multiple levels of superimposed structure has been a major theoretical stumbling block in composite materials. Here, we apply a new approach, the unified equation, to sort out the morphological details of these complex materials.

This article will

1. give a general background for morphological studies of CB composites using smallangle scattering;

2. give an overview of the unified equation, used to fit the data, and develop a new framework for application to polydisperse particulate systems;

3 . discuss the results from low concentration carbon black-high-density polyethylene (CB-PE) samples, and their thermal cycling, where the high $q$ scattering can be described in terms of the matrix and carbon contributions;

4. discuss the results from CB-poly(methyl metacrylate) (PMMA) composites to elucidate the carbon contribution to the high $q$ scattering in (3) using an amorphous matrix polymer; and

5. discuss the results from high-concentration CB-PE composites and their thermal cycling, which display a power-law-1 regime (for one-dimensional objects) reflecting linear agglomeration of $\mathrm{CB}$ aggregates.

\section{Background}

Carbon black-high-density polyethylene (CB-PE) composites have been used as self-resetting fuses for a number of years. ${ }^{1-3}$ In these composites, $\mathrm{PE}$ is crosslinked using $\gamma$-radiation. Although in commercial use, the morphology of these composites has never been completely understood; for instance, the mechanism of conductivity reduction at high currents and their related high resistive temperatures is still a matter of some debate. ${ }^{3}$ There may be an interplay between the crystalline morphology of polyethylene in these composites and the organization of carbon black aggregates such that, at the melting point for $\mathrm{PE}$, the carbon black aggregates no longer display sufficient connectivity to insure a conductive pathway through a film of the composite. The origins of this idea is the coincidence between the melting point of polyethylene and the resistive temperature of reduced conductivity. One mechanistic model for reversible conductivity involves voids at the lamellar interface of the $\mathrm{PE}$ crystallites. ${ }^{4}$ Wignall proposed the existence of these voids in a study using conventional pinhole SAXS measurements and a Debye-Bueche approach. This work on voids has recently been extended through absolute intensity measurements using SANS and SAXS with some success. ${ }^{5}$ Rather than voids, this article focuses on the possibility of local agglomeration of carbon aggregates as the source of switchable conductivity on size scales an order of magnitude larger than the proposed voids.

Furnace blacks can be described in terms of three levels of structure. ${ }^{6}$ On the smallest scale are primary particles, which are typically 200 to $1000 \AA$ in diameter. These primary particles aggregate and fuse in the furnace reactor into a second, larger level of structure. Aggregates can take a variety of forms from fumed-silica-like mass-fractal or linear structures to completely compact and roughly spherical domains. ${ }^{6}$ In the furnace black considered here, electron microscopy data indicate that the aggregates are dense, compact, and fairly spherical structures apparently composed of fused primary particles. The final, and largest, level of structure are agglomerates of these aggregates. Agglomerates are typically weakly bound and might be broken down into aggregates when a black is mixed with and dispersed in a polymer. All three of these structural levels are known to display a high degree of polydispersity. ${ }^{6}$ Typically, the polydispersity of aggregates is much broader than that of the primary particles, and the agglomerate polydispersity can vary widely depending on the mechanical history. In the carbon black studied here, the aggregates are composed of tightly packed primary particles much larger than the matrix polymer's (PE) lamellar-stacking period of about 250 $\AA$. This article presents morphological evidence that the physical properties of these carbon black-PE composites, such as their conductivity, may be predominantly understood in terms of the aggregate/agglomerate levels within them, and that the connectivity of these large structures may be associated with fiber bundles or larger structural features of crystalline PE (Fig. 1).

The carbon black used in this work contains aggregates with sizes on the submicrometer scale, the regime accessible using ultra-low- $q$ SAXS (or 


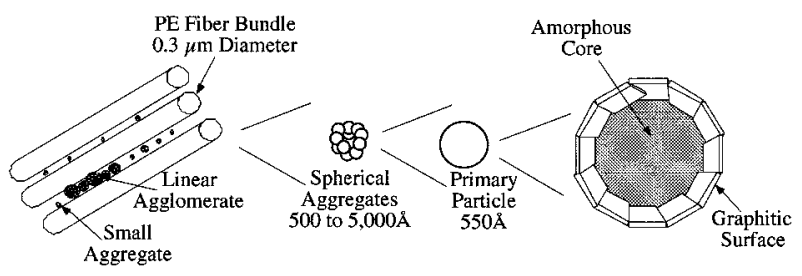

Figure 1. Schematic of structural levels in PE-carbon black composites, with smaller size scales from left to right. In this cartoon, linear agglomerates of CB aggregates (left) are associated with linear $\mathrm{PE}$ fiber bundles in the semicrystalline state.

SANS) instruments. These Bonse-Hart-type cam$\operatorname{eras}^{7}$ are available at several national facilities in the U.S.A., such as the National Institute of Standards and Technology instrument at Brookhaven National Laboratory ${ }^{7}$ and the Sandia National Laboratories instrument at the University of New Mexico. ${ }^{8}$ The Brookhaven instrument is capable of measurements up to roughly $1.4 \mu \mathrm{m}$ in radial size for a good scatterer, while the Sandia instrument, which is based on a rotating anode generator, is capable of measurements up to about $0.8 \mu \mathrm{m}$ radius. Both instruments yield smeared data on an absolute intensity scale that overlap with absolute intensity data from Oak Ridge National Laboratories' pinhole SAXS instrument ${ }^{9}$ after desmearing, (cf. Fig. 7, discussed below). Recently, Wignall and Agamalian ${ }^{10}$ have developed a similar Bonse-Hart instrument for SANS at Oak Ridge National Laboratory.

Data from these facilities can be combined with pinhole SAXS data since they overlap by up to a decade in scattering vector $q$, where $q$ $=\frac{4 \pi}{\lambda} \sin \left(\frac{\theta}{2}\right), \theta$ being the scattering angle. SAXS data can be further extended at high- $q$ by combination with X-ray diffraction (XRD) data. BonseHart data also overlap with static small-angle light scattering data (SALS) ${ }^{11}$ at low- $q$. The latter is, however, not an option for these CB composites due to their complete absorption of visible light. Combined X-ray data can cover up to six orders in $q$ (cf. Fig. 7), yielding a full picture in $q$-space of a material's morphology.

\section{The Unified Approach to SAXS}

Such wide $q$-range data describe microstructural features on many length scales. Until recently, a scattering theory, which could account for the overlap of these morphological features in a general way, was not available. Because of the need for a global description of scattering data, the unified approach was developed. ${ }^{11-13}$ The unified equation describes material microstructure in terms of the levels of structure. Each level of structure corresponds to a Guinier regime, ${ }^{14}$ combined with a structurally limited power-law regime. The associated power-law serves to describe the integral properties of the structure, the size, and composition of which are described by the Guinier regime. For example, a surface-fractal particle's integral properties are related to the surface roughness described by the surface-fractal dimension $d_{s}$ and the surface-to-volume ratio $S / V$. For a two-dimensional (2-D), smooth, sharp surface, the power-law follows Porod's law ${ }^{14}$ and the slope of a $\log I$, intensity, versus $\log q$, scattering vector, plot is -4 . For a fractally rough surface, the power-law slope is somewhat shallower than -4 following a slope of $\left(d_{s}-6\right)$, as described by Bale and Schmidt. ${ }^{15-18}$ For a single structural level, the unified equation is given by

$$
I(q)=G \exp \left(\frac{-q^{2} R_{g}^{2}}{3}\right)+B q_{\text {limited }}^{-P}
$$

where $q_{\text {limited }}$ structurally limits the power-law at low- $q$, near $R_{g}$, and is given by

$$
q_{\text {limited }}=\frac{q}{\left[\operatorname{erf}\left(\frac{q R_{g}}{\sqrt{6}}\right)\right]^{3}}
$$

$G$ is called the Guinier prefactor, ${ }^{14} R_{g}$ is the radius of gyration, and $B$ is the power-law prefactor, described by the regime in which the power-law slope $-P$ falls. As noted above, for surface fractals, $P$ is $\left(6-d_{s}\right)$, and $d_{s}$ is the surface fractal dimension. The two components of scattering for a single structural level, represented by the two terms of eq. (1), are easily distinguished in a $\log I$ versus $\log q$ plot. The Guinier term, the first term in eq. (1), dominates scattering at low $q, q R_{g} \leq 1$, and appears as a knee while the power-law, the second term, appears as a line at high- $q, q R_{g}$ $\geq 3$, for example, in the fit of Figure 4 .

\section{Power-Law Distributions in SAXS}

In addition to the fractal description above, it is also possible to obtain a wide regime in $q$ of surface-fractal-like scaling, that is, the power-law slope between -3 and -4 , in the absence of particles with self-similar surface roughness. For ex- 
ample, a power-law polydispersity in particle size over a wide range of size would lead to such a scattering pattern. The scattering at low- $q, q R_{g}$ $\ll 1$, from eq. (1), is given by $G$ of eq. (3), below. $G$ is typically several orders of magnitude larger than the higher- $q$ scattering in the Guinier and power-law regimes. As a first approximation, the scattering by a polydisperse sample can be estimated as a sum of $G$ terms. For a given particle radius, $G$ in eq. (1), is given by

$$
G=N \Delta \rho_{e}^{2} V_{\text {particle }}^{2}
$$

for a dilute suspension of monodisperse particles of excess electron density $\Delta \rho_{e}$, volume $V_{\text {particle, }}$, and number $N$ in the scattering volume. Under dilute conditions, the contribution to the scattering pattern of each size particle $R_{g i}$ in a polydisperse system can be considered independently and summed. If the particles are spheres, the particle radius $R$ is given by $\sqrt{\frac{5}{3}} R_{g}$. The number distribution depends on a binning size $\Delta R$ and an incremental change in the particle number, $\Delta N /$ $\Delta R$, so eq. (3) for a polydisperse sample is written as

$$
\sum G=\left(\Delta \rho_{e}\right)^{2} \sum \frac{\Delta N}{\Delta R} \Delta R(V(R))^{2}
$$

In this way, a sum of scattering functions can be approximated for a polydisperse sample, where $V_{\text {particle }, i}$ scales as $R_{i}^{3}$. Given a constant particulate excess electron density and a continuous function for $N(r)$, this sum of equations, of the type of eq. (3), can be replaced by the integral,

$$
I_{\text {Polydisperse }} \cong \int \frac{d N(r)}{d r}\left(\Delta \rho_{e}\right)^{2}\left[\frac{4 \pi r^{3}}{3}\right]^{2} d r
$$

for spherical particles. Assuming a power-law particle size distribution function,

$$
N(r)=C r^{-m} \text { and } \frac{d N(r)}{d r}=-m C r^{-m-1}
$$

where $C$ and $m$ are constants, the observed intensity for a power-law polydisperse sample of spherical domains in the power-law scattering regime is approximated by

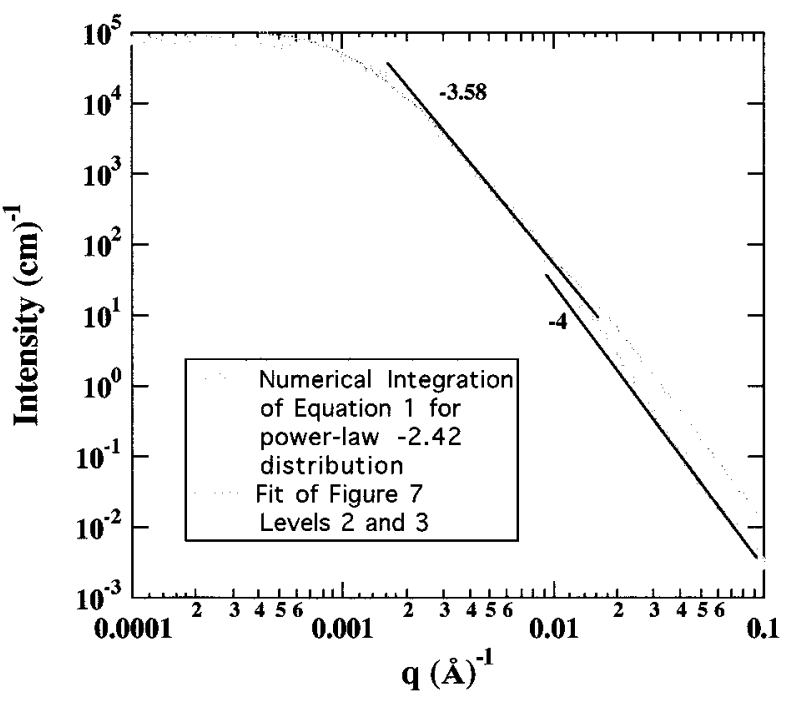

Figure 2. Numerical integration of eq. (1) for the parameters of Figure 8.

$$
\begin{gathered}
I_{\text {Polydisperse }}(R) \cong m C\left(\Delta \rho_{e}\right)^{2}\left(\frac{4 \pi}{3}\right)^{2} \int r^{5-m} d r \\
=\frac{m C\left(\Delta \rho_{e}\right)^{2}}{(6-m)}\left(\frac{4 \pi}{3}\right)^{2} R^{6-m} \\
I_{\text {Polydisperse }}(q) \cong \pi^{6-m} \frac{m C\left(\Delta \rho_{e}\right)^{2}}{(6-m)}\left(\frac{4 \pi}{3}\right)^{2} q^{m-6}
\end{gathered}
$$

where $6-m=P$ from eq. (1). For surfacefractal-like scaling, $3<P \leq 4$, and, $3>m \geq 2$. In eq. (7), Bragg's law was used to approximate the radial size, $R, q=\pi / R$.

Figure 2 shows the result of a more exact numerical integration of eq. (1) for dilute spheres with sharp interfaces (displaying Porod scattering) and a power-law size-distribution of $m$ $=2.42$ [eq. (6)]. The limits to the power-law distribution were taken from the fit of Figure 8, levels 2 and 3, which will be discussed in detail below. The power-law scattering slope agrees with eq. (7). Although the fit to Figure 8, reproduced in Figure 2, and the integral do not agree at high- $q$, Figure 2 clearly demonstrates that a power-law size distribution can lead to surfacefractal-like scaling in scattering. The excess intensity at high- and low- $q$ may be related to deviations from a power-law distribution.

A power-law particle size distribution could result from a number of natural phenomena. For example, if the probability of breakup of domains 
in a grinding process were proportional to the surface area of the grains, a power-law -2 distribution might be expected, which would appear as a Porod-like power-law regime, -4 slope. Such a surface-dominated decomposition process might describe the microstructure of some common materials, such as beach sand. In a growth process where surface charges lead to repulsion of liquid domains, growth of large domains might be hindered in proportion to their surface area, also leading to number distributions scaling as $r^{-2}$. For growth or decomposition processes limited by volumetric considerations, such as particle mass, a power-law scattering of slope -3 might be observed. This is important since power-laws of slope -3 are forbidden by monodisperse surface or mass-fractal laws. It is easy to envision a growth process effected by a combination of surface and volumetric effects, which would lead to an apparent surface-fractal scattering law. In fact, such power-law particle size distribution functions have been proposed for the growth of carbon black aggregates at high temperatures in furnace reactors. ${ }^{19-25}$ For example, Bansal and Donnet ${ }^{19}$ review an aggregate growth mechanism based on surface deposition, $N(r)$ scaling as $r^{-2}$, and volumetric coalescence, which might lead to surface-fractal-like power-law distributions over a wide range of size. In addition to aggregate growth in a furnace reactor, consideration should be given to subsequent oxidation of aggregates, which may be governed by surface terms. ${ }^{19,26-30}$

Particle size distributions in carbon black are often described in terms of a heterogeneity index. ${ }^{6}$ For power-law distributions, the heterogeneity index, ${ }^{31} H I$, can be calculated according to $H I=($ mass average size $) /($ number-average size). Under the assumption that the scaling regime is large, that is, close to a decade in size $\left(R_{\max }\right.$ $\gg R_{\text {min }}$,

$$
H I=\frac{(P-3)(P-5)}{(P-2)(P-6)} \frac{R_{\max }}{R_{\min }},
$$

where $P$ is the negative of the scattering powerlaw slope, and $R_{\max }$ and $R_{\text {min }}$ are the maximum and minimum sizes for the scaling regime in scattering. For many carbon blacks, HI for aggregates is typically $2.0 \pm 0.1^{6}$ before mixing with a polymer. $P$ from the surface-fractal-like scattering regime for furnace blacks is typically $3.6 \pm 0.05$ with the ratio of $R_{\text {max }}$ to $R_{\text {min }}$ being close to 9 (using the limiting $R_{g}$ 's from scattering or micros- copy data for the diameter, $D$ ). It can be seen that there is at least a coincidental agreement between the prediction of eq. (8), and the microscopically determined $H I$, lending support to the polydisperse aggregate approach.

A scattering curve such as that in Figure 2 displays two levels of structure, that is, two power-law regimes and two Guinier regimes. To model such a scattering profile, eq. (1) can be extended to describe any number of structural levels if a sufficient range of $q$ is observed in the scattering, ${ }^{11-13}$

$$
\begin{aligned}
I(q) \approx \sum_{i=1}^{n}( & G_{i} \exp \left(\frac{-q^{2} R_{g, i}^{2}}{3}\right) \\
& \left.\quad+B_{i} \exp \left(\frac{-q^{2} R_{g,(i-1)}^{2}}{3}\right)\left(\frac{1}{q_{i, \text { iimited }}}\right)^{P_{i}}\right)
\end{aligned}
$$

where the summation is over the number of structural levels in a material $n$, and $q_{i \text {,limited }}$ is given by eq. (2) using $R_{g, i}$. A structural level in scattering is composed of a structural size reflected in the first term and a power-law scaling regime, as reflected in the second term. Here, level 1 is the smallest structural level. Equation $1^{\prime}$ presents four free parameters for each level of structure in the most general case, as follows: the contrast factor $G_{i}$; the radius of gyration $R_{g, i}$; the powerlaw prefactor $B_{i}$; and the power-law slope $-P_{i}$. Some of these parameters can be calculated according to morphological models for the system and conventional scattering laws, such as Porod's Law and scattering laws for surface and massfractals or other relationships, such as the polydispersity law described here. The appearance of $R_{g(i-1)}$, high- $q$ power-law limit, in the second term implies that level $i$ is composed of the smaller level $(i-1)$; that is the power-law regime for level $i$ is structurally limited at $R_{g(i-1)}$. If the two levels are not associated, then a free parameter can be used in place of $R_{g(i-1)}$, or $R_{g(i-1)}$ can be set to 0 for an unobservable limit to the low- $q$ power-law. ${ }^{11-13}$

Since the power-law-polydispersity integration, shown in Figure 2, leads to two power-law regimes and two associated Guinier regimes, the integration of Figure 2 can be modeled with two structural levels just as a surface fractal structure could be modeled with two levels. ${ }^{11-13}$ The primary particles of the carbon black aggregates are always observed to display a -4 power-law scaling so $P_{1}=4$ and $B_{1}=2 \pi G_{1} S_{\text {primary }}$ 
particle/ $\left(V_{\text {primary particle }}\right)^{2} . G_{1}$ is obtained from eq. (3) for the primary particles. In the lower- $q$ surface-fractal-like regime, $P_{2}=6-m$ from the power-law polydispersity relationship and $B_{2}$ is approximated by eq. (7), $R_{g, 2}$ for the largest level corresponds to the largest sized particles. $G_{2}$ for the largest level is a summation of $G$ terms for all of the particles in the power-law distribution,

$$
G_{2} \approx \frac{m C\left(\Delta \rho_{e}\right)^{2}}{(6-m)}\left(\frac{4 \pi}{3}\right)^{2}\left(\frac{5}{3}\right)^{\frac{(6-m)}{2}} R_{g, 2}^{(6-m)}
$$

for spherical domains and a power-law distribution over close to a decade.

If agglomerates of aggregates are observed in the scattering pattern, an additional structural level may be necessary at low-q. Typically, agglomerates display a linear or ramified morphology, leading to a weakly sloped, mass-fractal, or low-dimensional structural regime in scattering. For instance, if the agglomerates are rods, as in Figure 1, then the surface-fractal-like scaling from a power-law polydispersity regime would decay at low- $q$ to a regime of slope -1 . For rods, $P$ $=1$ and $B$ is given by, ${ }^{11-14}$

$$
B_{\text {rod regime }} \approx \frac{\pi G_{\text {rod regime }}}{L}
$$

where $L$ is the rod's length.

Scattering from most carbon blacks is well described by this unified approach down to the size of the substructure of the primary particles. This substructure is sometimes observed as a powerlaw -2 regime at highest $q$ in the SAXS regime, which may be related to a partially graphitic character for certain grades of carbon black and for graphitized carbon black ${ }^{6,32}$ (Fig. 1). This graphitic regime shows a platelet-like morphology and can be modeled using an extension of eq. ( $\left.1^{\prime}\right)$ for an additional platelet structural-level. ${ }^{33}$ As mentioned above, low-dimension objects, such as platelets, scatter according to power-law of $-d_{f}$ slope ${ }^{17}$ where $d_{f}$ is the object's dimension. $d_{f}$ is 2 for 2-D graphitic layers.

\section{Concentration Effects}

The above discussion assumes that the carbon aggregates and primary particles are in dilute concentrations and that interparticle interference can be ignored. As a first approximation, higher concentrations, in the absence of a correlation peak in the scattering, can be understood through a redefinition of the Guinier prefactor, $G_{\text {conc }}$,

$$
G_{\mathrm{conc}}=\phi(1-\phi) N \Delta \rho_{e}^{2} V_{\text {particle }}^{2}
$$

where $\phi$ is the local volume fraction of domains. (More detailed consideration of interference is not justified in these data sets since a clear correlation peak is not observed.) Thus, for increased volume fractions, a depletion of the scattered intensity is expected. The power-law prefactor $B$, due to surface scattering (either fractal or smooth) should remain unchanged with changes in packing; that is, $B$ is not effected by interparticle interference [Guinier and Fournet, ${ }^{14}$ (page 80)]. $B$ will change in proportion to the square of contrast changes and linearly with concentration. For a surface-fractal-like scaling regime, which is due to polydispersity in particle size, changes can occur in $B$ with interparticle interference depending on how packing changes with particle size. Equation ( $\left.3^{\prime}\right)$ can be used to approximate the local concentration of carbon black aggregates in a composite sample with nonuniform aggregate distribution (Fig. 1). The depletion in scattered intensity described by eq. $\left(3^{\prime}\right)$ is due to interparticle interference and is expected to reflect concentration on the size scale of the domain, that is, the local concentration of particles. In a system with coalescence of particles, eq. $\left(3^{\prime}\right)$ can give a measure of the local concentration enhancement due to agglomeration.

\section{EXPERIMENT AND DISCUSSION}

Scattering experiments were performed on compression-molded plaques having thicknesses in the range of $0.25 \mathrm{~mm}$. The polymer and carbon blends were milled in a 50-g Brabender mixer until a plateau in the torque was observed (about 20 min). 1-mL-thick plaques were made from these mixtures using a heated Carver press at about $180^{\circ} \mathrm{C}$. CB-PE composites were fabricated using commercially available linear, high-density polyethylene (HDPE) having a very low degree of branching. This material has a $\bar{M}_{w}=145,000 \mathrm{~g} / \mathrm{mol}$ and $\bar{M}_{n}=20,000 \mathrm{~g} / \mathrm{mol}$, with $\rho=0.96 \mathrm{~g} / \mathrm{cm}^{3}$. Sample 29-8 is composed of 5\% by volume CB in HDPE, sample 29-4 is composed of $35 \%$ by volume $\mathrm{CB}$ in HDPE. Plaques were also made using a standard commercial grade of noncrystalline 


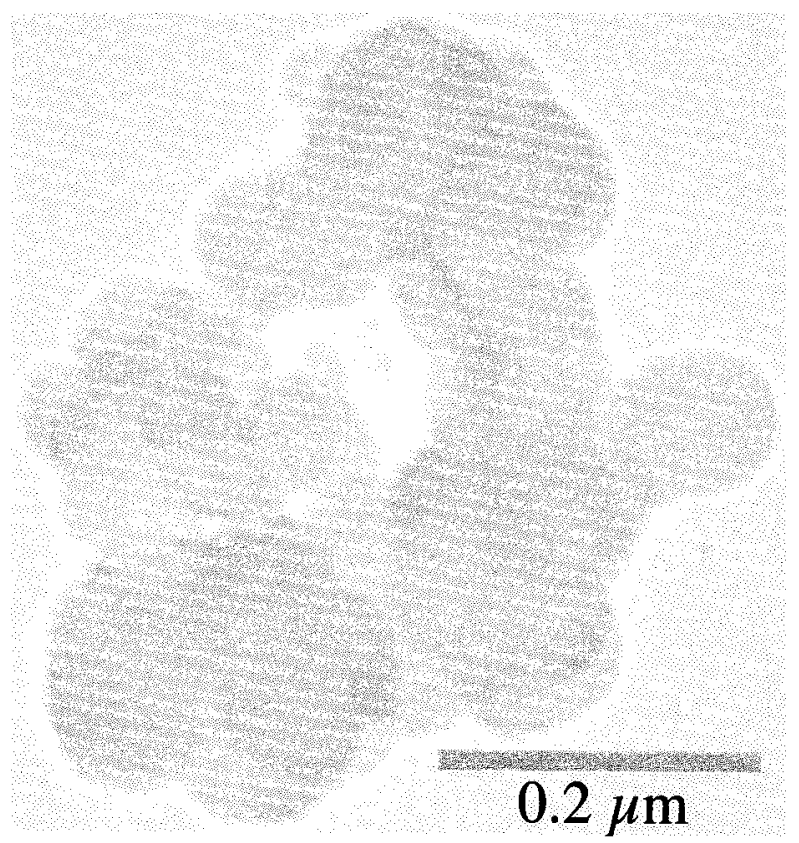

Figure 3. Transmission electron microscopy micrograph of a single agglomerate composed of aggregates of the carbon black used in this study. The agglomerates are broken up into fairly isolated aggregates on milling with PE (or PMMA). The carbon consists of a high degree of aggregate size polydispersity.

PMMA as the matrix material in order to understand the nanoscale structure of the carbon black in the absence of matrix nanoscale features.

The black used in this work is a commercially obtained specialty carbon black made by the furnace process. It has a mean primary particle size of nominally $\sim 800 \AA$ diameter. Figure 3 shows a typical agglomerate of $\mathrm{CB}$ aggregates for this black. Agglomerates are largely broken up when milled with a high-viscosity polymer, leading to a highly polydisperse dispersion of aggregates. The aggregate polydispersity is evident in Figure 3. This single carbon black is used exclusively in this series of measurements and is of intermediate structure having a DBP number of $\sim 80$ [see Herd $^{6}$ (page 133) and other literature $\left.{ }^{34}\right] . \mathrm{N}_{2}$ absorption on this black indicates little or no porosity, supporting the idea that the carbon black aggregates have smooth surfaces. As mentioned above, polydispersity in size for the various levels of structure in carbon blacks is characterized by the heterogeneity index (HI) of eq. (8). The aggregate $H I$ for the black used in these experiments is close to 2 according to microscopy measurements. The HI is calculated from the ratio of the mass average and number-average sizes using measurements obtained from micrographs, such as Figure 3. An HI of 2 reflects a typical broad distribution in aggregate size. Microscopy data indicate that the aggregate diameters span close to a decade in size. As mentioned above, there is a coincidence for most furnace blacks that a powerlaw close to -3.6 is observed in SAXS spanning close to a decade in size and that microscopy generally shows a polydispersity of aggregate size over close to a decade with $H I$ close to 2 for many furnace blacks. This coincidence supports the view that the source of the surface-fractal-like power-law scattering is polydispersity in aggregate size [eq. (6)].

\section{5\% Carbon Black-PE Composites}

A typical combined SAXS curve for a low concentration ( $5 \%$ by volume, $\rho_{C B}=1.9 \mathrm{~g} / \mathrm{cm}^{3}$ ) CB-PE composite displays easily resolved features which relate to independent contributions from the aggregates of carbon black on a submicrometer scale and the lamellar morphology of the crosslinked $\mathrm{PE}$ on a nanometer scale. In Figure 4, eq. (1) was used to fit the data from 0.0007 to about $0.01 \AA^{-1}$, and this fit was extrapolated over the entire data range. The fit indicates that the carbon black aggregates are adequately described by a surfacefractal-like power-law scattering regime with $P$

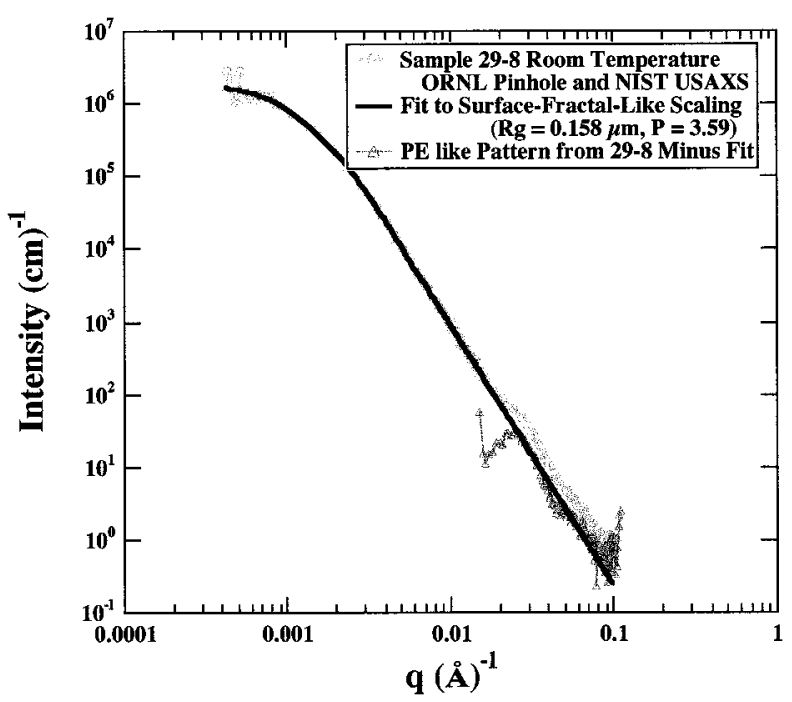

Figure 4. Typical USAXS and SAXS data from a CB-PE composite of $5 \%$ by volume CB loading at room temperature. Fit uses eq. (1) and only the data between 0.0007 and $0.01 \AA^{-1}$. The fit has been extended over the entire range for comparison. 
Table I. Fit Parameters and Calculations for All Samples

\begin{tabular}{|c|c|c|c|c|c|c|}
\hline Sample & $R_{\phi}(\AA)$ & $G\left(\mathrm{~cm}^{-1}\right)$ & $B\left(\mathrm{~cm}^{-1} \AA^{-P}\right.$ & $P$ & Agr $\mathrm{Hl}^{\mathrm{e}}$ & Calc I Ratio ${ }^{f}$ \\
\hline $5 \% 200^{\circ} \mathrm{C} \mathrm{PE}$ & $1540 \pm 30$ & $3.09 \pm 0.07 \times 10^{6}$ & $7.2 \pm 0.3 \times 10^{-5}$ & $3.59(\mathrm{~F})$ & 1.68 & \\
\hline $5 \%$ Cooled PE & $1580 \pm 30$ & $1.86 \pm 0.04 \times 10^{6}$ & $5.9 \pm 0.2 \times 10^{-5}$ & $3.59 \pm 0.01$ & 1.72 & \\
\hline Ratios $200^{\circ} \mathrm{C} /$ Cooled & 0.975 & 1.66 & 1.2 & & & $1.26\left(\phi_{C r}=0.96\right)^{\mathrm{g}}$ \\
\hline 1\% PMMA & $1603 \pm 10$ & $1.15 \pm 0.04 \times 10^{5}$ & $4.6 \pm 0.2 \times 10^{-6}$ & $3.58 \pm 0.01$ & 1.74 & \\
\hline $35 \%$ PMMA $(\text { Agr })^{\mathrm{a}}$ & $760 \pm 5$ & $5.84 \pm 0.04 \times 10^{5}$ & $1.5 \pm 0.1 \times 10^{-4}$ & $3.58(\mathrm{~F})^{\mathrm{d}}$ & & \\
\hline $35 \%$ PMMA $(\mathrm{Agl})^{\mathrm{b}}$ & $1650 \pm 30$ & $3.09 \pm 0.05 \times 10^{5}$ & $3.3 \pm 0.3 \times 10^{-2}$ & $1(\mathrm{~F})$ & & \\
\hline Ratios $35 \% / 1 \%$ & 0.474 (Agr) & $7.77(\mathrm{Agr}+\mathrm{Agl})$ & 39 (Agr) & & & 7.96 \\
\hline $35 \% 200^{\circ} \mathrm{C} \mathrm{PE}(\mathrm{Agr})$ & $475 \pm 5$ & $7.11 \pm 0.04 \times 10^{5}$ & $1.2 \pm 0.1 \times 10^{-4}$ & $4(\mathrm{~F})$ & & \\
\hline $35 \% 200^{\circ} \mathrm{C} \mathrm{PE}(\mathrm{Agl})$ & & & $1390 \pm 50$ & $1.09 \pm 0.01$ & & \\
\hline $\begin{array}{l}35 \% \mathrm{Cl} \text { and } R T \\
\text { Runs PE }(\mathrm{Agr})^{\mathrm{c}}\end{array}$ & $536 \pm 4$ & $4.00 \pm 0.03 \times 10^{5}$ & $6.5 \pm 0.1 \times 10^{-5}$ & $4(\mathrm{~F})$ & & \\
\hline $\begin{array}{l}35 \% \mathrm{Cl} \text { and } R T \\
\text { Runs } \mathrm{PE}(\mathrm{Agl})\end{array}$ & & & $802 \pm 30$ & $1.07 \pm 0.01$ & & \\
\hline Ratios $200^{\circ} \mathrm{C} /$ Cooled & 0.886 & 1.78 & 1.85 (Agr) 1.73 (Agl) & & & $1.26\left(\phi_{C r}=0.80\right)$ \\
\hline $\begin{array}{l}\text { a Agr denotes agg } \\
\text { b Agl denotes aggl } \\
{ }^{\mathrm{c}} \text { Cl denotes coolec } \\
{ }^{\mathrm{d}} \mathrm{F} \text { denotes fixed } \\
{ }^{\mathrm{e}} \text { Agr HI denotes } \\
{ }^{\mathrm{f}} \text { Calc } I \text { Ratio den } \\
{ }^{\mathrm{g}} \phi_{C r} \text { denotes effe }\end{array}$ & $\begin{array}{l}\text { gates. } \\
\text { merates. } \\
R T \text { denotes } \\
\text { arameter. } \\
\text { es calculated } \\
\text { ive } \phi \text { in cryst }\end{array}$ & $\begin{array}{l}\text { om temperature. } \\
\text { ogeneity index using } \\
\text { ntensity ratio. }\end{array}$ & $R_{g, \min }=200 \AA$ & & & \\
\hline
\end{tabular}

$=3.59 \pm 0.006$. The fit parameters from eq. (1) are given in Table I, "5\% Cooled PE." Absolute intensity values ( $G$ and $B$ ) typically have a confidence margin of $10 \%$ due to the probable errors in sample thickness. The standard deviation values in Table I are obtained by propagation of the statistical error in the data, from $\sqrt{\text { Counts, }}$ through the least-squares fits using eqs. (1) or $\left(1^{\prime}\right)$.

The low- $q$ fit from the CB aggregates in Figure 4 can be subtracted from the composite data to elucidate the features at high- $q$ under the assumption of independent contributions to the scattered intensity for the low- and high- $q$ features. This difference data (triangles in Fig. 4) are close to the expected curve for lamellar high-density PE (HDPE) of similar crystallinity. Pinhole SAXS data from a typical HDPE sample are compared with this difference curve in Figure 5. Both curves in Figure 5 are in absolute intensity (i.e., no offset was used; absolute intensity is divided by the irradiated volume to yield units of $\mathrm{cm}^{-1}$ ). Figure 5 demonstrates that the high- $q$ oscillations in the pinhole SAXS curve are due solely to lamellar scattering from the PE component of the composite. Similar fits and subtractions have been done for a series of these composites with identical results. The statistical error is higher for the higher concentration CB composites. There is no indication of a reduction of $\mathrm{PE}$ crystallinity with CB loading from these curves.

As noted above, there are at least two models that could explain the apparent surface-fractal

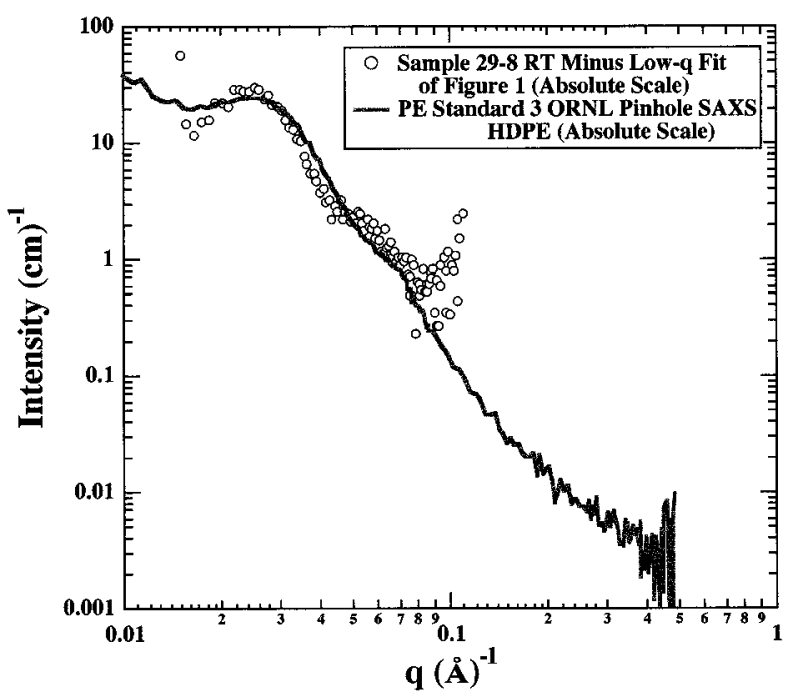

Figure 5. Comparison on an absolute scale of similar HDPE pinhole SAXS data with the difference between the fit and the data for the PE-CB composite of Figure 1 . 


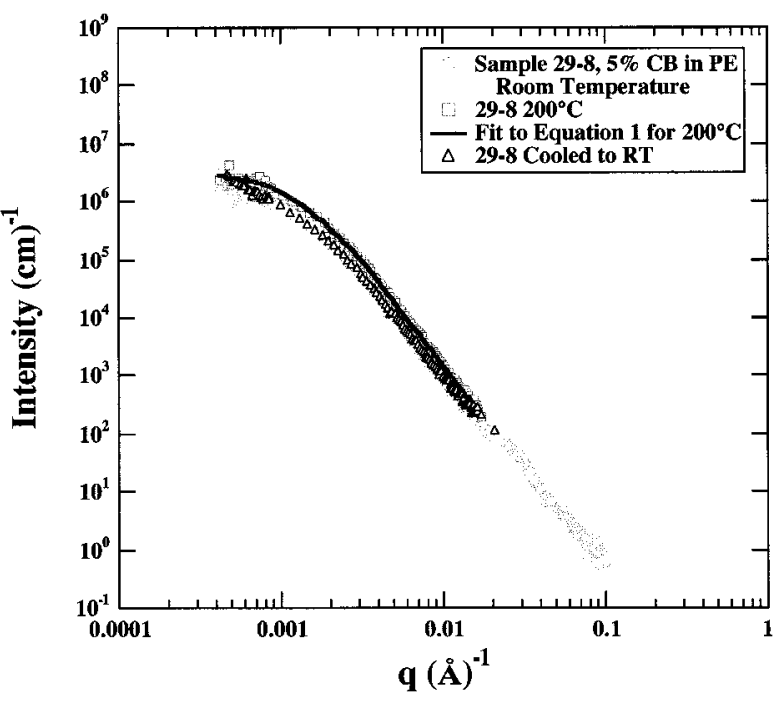

Figure 6. Heating and cooling of sample 29-8, 5\% CB in crosslinked PE matrix. Fit is to eq. (1) for the $200^{\circ} \mathrm{C}$ run.

scaling regime for the carbon black aggregates at low- to intermediate- $q$, as follows: (1) a surfacefractal model based on fairly rough aggregates, with surface fractal scaling extending over close to a decade in size. This model is disputed by the low porosity of the carbon black aggregates and by microscopy data, which show no apparent surface roughness; (2) a power-law aggregate size distribution, as discussed above, and supported by microscopy and the $H I$ calculation.

A completely reversible change occurs in the observed scattering patterns on heating above the melting point of the PE matrix (Fig. 6). The fit parameters to eq. (1) for the data from the $200^{\circ} \mathrm{C}$ run in Figure 6 are given in Table I. The power-law exponent $P$ is fixed at the value from Figure 2 in the fit of Figure 6 . The main statistical difference between the room temperature crystalline $\mathrm{PE}$ scattering data and $200^{\circ} \mathrm{C}$ amorphous PE scattering data pertain to the Guinier prefactor, $G, G_{\text {figure } 5} / G_{\text {figure 3 }}=1.66$ (third row in Table I). This change may be related to the change in contrast since the electron density of the PE matrix decreases on melting. A calculation of this contrast change, using eq. (3), yields a $G$ ratio greater than 1, namely, 1.26 in Table $\mathrm{I}$ (using $\rho_{C B}=1.95 \mathrm{gm} \mathrm{cm}^{3}, \rho_{\mathrm{PE}, \mathrm{amo}, 200^{\circ} \mathrm{C}}=0.840$ $\mathrm{gm} \mathrm{cm}^{3}$, and $\rho_{\mathrm{PE}, \mathrm{cry}, R T}=0.960 \mathrm{gm} \mathrm{cm}^{3}$ ) but does not completely account for the observed change.

A possible explanation for the observed $G$ ratio might be drawn from the plausible morphological changes, which occur on crystallizing the PE ma- trix (Fig. 1). The PE long-period peak in Figure 5 indicates that, on a nanoscale, $\mathrm{PE}$ lamellae (close to $175 \AA$ in thickness using $\approx 70 \%$ crystallinity) correlate at a distance of about $250 \AA$. These nanosized features are well below the size scale of either the aggregates or primary particles of this carbon black. On a larger size scale, these stacked lamellae are typically grouped into fiber bundles of submicron diameter (typically 0.1 to $0.3 \mu \mathrm{m}$ diameter), which radiate from a nucleation site (Fig. 1). Larger carbon black aggregates are probably excluded from these fibers, which account for the majority of the polymer component since the $\mathrm{PE}$ is about $70 \%$ crystalline. Exclusion from larger scale structures, such as spherulites (typically 10 to $100 \mu \mathrm{m}$ ) is also possible. In any case, it might be expected that the local volume fraction of carbon black is significantly enhanced, even in the 5\% sample, especially for the larger aggregates, explaining the anomalous decrease in $G$ on cooling. The local volume fraction of $\mathrm{PE}$ can be calculated from eq. (3') under the assumption that this is the sole source of intensity depletion in the crystalline sample and that the carbon black is randomly arranged in the amorphous $\mathrm{PE}$ sample, $\phi_{\text {amo }}=0.05$,

$$
\frac{G_{\mathrm{amo}}}{G_{\text {cryst }}}=\frac{\phi_{\mathrm{amo}}\left(1-\phi_{\mathrm{amo}}\right)(\Delta \rho)_{\text {amo }}^{2}}{\phi_{\text {cryst }}\left(1-\phi_{\text {cryst }}\right)(\Delta \rho)_{\text {cryst }}^{2}}
$$

yielding $\phi_{\text {cryst }}=0.96$ (assuming that the local $\phi$ can only increase from a random distribution). The observed intensity change is consistent with significant exclusion of carbon black from the PE fiber bundles (Fig. 1). An apparent increase in the local large-aggregate concentration to $96 \%$ could account for an increase in conductivity in the crystalline matrix; however, such a large increase might seem somewhat unreasonable since it would require the transport of large aggregates over relatively large distances without structural modification.

The ratio of power-law prefactors $\left(B_{200^{\circ} \mathrm{C}}\right)$ $\left.B_{R T}\right)_{\text {measured }}=1.23$ (Table I) is close to the predicted intensity ratio, 1.26, accounting only for matrix contrast changes on melting. The surface fractal model agrees with this behavior. For the polydisperse aggregate model, the difference between the observed $G$ and $B$ ratios can be explained if the smaller aggregates in the powerlaw scaling regime see less or no enhancement of their concentration since they are smaller or comparable to the PE fiber bundle diameter and may 


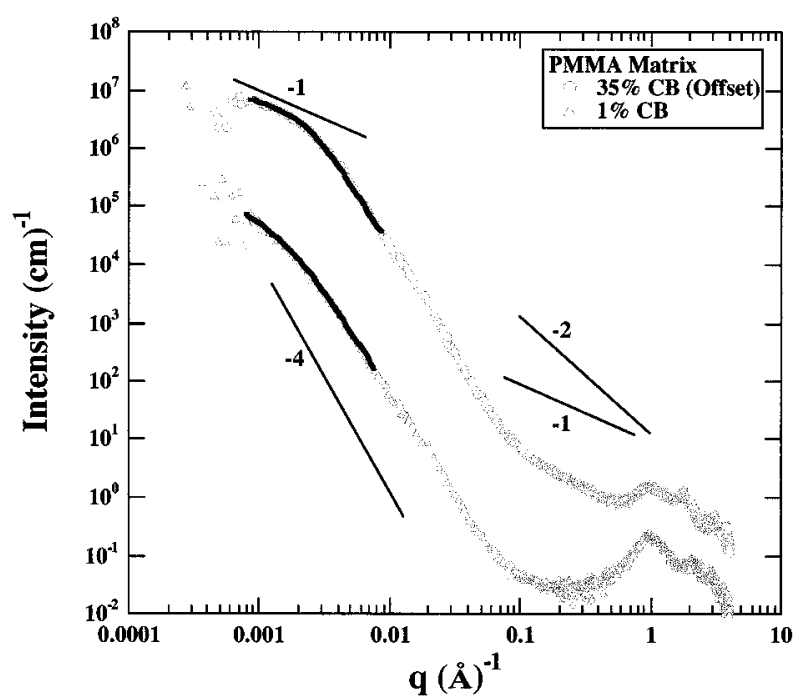

Figure 7. $\mathrm{CB}$ in amorphous PMMA matrix. Fit to $1 \%$ data uses eq. (1) and low- $q$ parameters from Figure 8 (subscript 3 parameters). $35 \%$ data have been offset (multiplied by 7) in intensity. 35\% data are fit with a two-level fit (aggregate and agglomerate) with $P_{\text {aggregate }}$ fixed at the value for the $1 \%$ sample.

be partly incorporated in the lamellar stacking for the smallest-sized aggregates (Fig. 1), especially at low concentrations.

An alternative explanation to eq. (11) for the enhancement of low- $q$ intensity is found in the higher concentration composites (Fig. 10), discussed below. In these composites, there is support for a model based on break up of the larger carbon black aggregates, on mixing with $\mathrm{PE}$, followed by reagglomeration into fairly linear clusters of aggregates in the PE, fiber-bundle matrix (Fig. 1). If the aspect ratio for these linear agglomerates is less than about 2, scattering data will not resolve the question of whether there is a linear scaling regime, ${ }^{35}$ that is, for the $5 \%$ room temperature run. In such a case, the two sizes to a linear agglomerate, the length and diameter, would appear as a single Guinier knee, with an enhanced intensity at low- $q$. For low aspect ratios, eq. (11) is sufficient to approximate these intensity changes.

\section{Carbon Black-PMMA Composites}

Since the nanoscale structure of carbon black is obscured in the PE composites by the PE lamellae (Fig. 5) 1 and 35\% carbon black samples in an amorphous PMMA matrix were examined using the same approach (Fig. 7). PMMA has a lower melt viscosity than PE; hence, the PMMA composites were subjected to a weaker shear history when compared with the PE samples. It was expected that this might alter the submicrometer morphology (low- $q$ ) but would not significantly effect the nanometer scale structure. The surfacefractal-like scaling regime for the 5\% carbon black-PE and $1 \%$ carbon black-PMMA samples has similar slope, indicating either that the surface roughness or that the polydispersity scaling are not effected by the change in matrix polymer (Table I). It seems reasonable to assume that features smaller than this scaling regime (higher- $q$ ) are also not effected by the change in matrix polymer.

As noted above, the low- $q$ knee in Figure 7 corresponds to the largest aggregate radius of gyration under a polydisperse aggregate model. This Guinier-knee shifts to higher $q$ in the $35 \%$ sample, indicating smaller aggregates or a truncation of the dispersion in size at the largest sizes. This is consistent with break up of large aggregates in the higher concentration sample. The power-law regime for the $35 \%$ sample can be fit with $P$ from the $1 \%$ sample but is limited in range due to the reduction in the large-scale limit to aggregate scaling (at low $q$ ). The slope in Figure 7 for this low- $q$ regime might be close to -4 if a sufficient range were available. A slope of -4 would be consistent with surface dominated aggregate break up; that is, $m=2$, as discussed above. In the $35 \%$ carbon black-PE composites discussed below, such a power-law -4 regime seems to be supported.

In the 35\% curve of Figure 7, there is weak evidence at lowest- $q$ for a low-dimensional scaling regime (line of -1 slope at lowest $q$ ). The low- $q$ Guinier regime cannot be fit reasonably in the absence of this component. A similar, but much more prominent, linear regime is observed for the $35 \%$ carbon black-PE samples shown below (Fig. 10 ). The power-law in the lowest- $q$, agglomerate regime was fixed to -1 because of this observation in the $35 \%$ carbon black-PE composites, although there are insufficient data in the 35\% carbon black-PMMA composite to determine this slope. Carbon black aggregates are known to agglomerate into low-dimensional mass-fractal structures. ${ }^{6}$ A model for the $35 \%$ scattering pattern is the break up of the larger aggregates in the higher concentration sample, followed by agglomeration in the shear field into fairly linear structures. The fit of the $35 \%$ sample uses two structural levels with the scaling regime for the 
agglomerate structure fixed at a rodlike -1 slope. The surface-fractal-like scaling regime is fixed to the power-law used for the $1 \%$ sample, -3.58 .

In Table I, eq. (11) has been used to calculate the expected intensity ratio between the $1 \%$ and $35 \%$ samples, $\left(G_{35 \%} / G_{1 \%}\right)_{\text {calculated }}=7.96$. The calculated value is within experimental error of the value obtained by summing the Guinier prefactor for the two levels of structure, in the $35 \%$ sample, and dividing by the Guinier prefactor for the $1 \%$ sample, $\left(G_{35 \%} / G_{1 \%}\right)_{\text {measured }}=7.77$. However, the ratio for the polydispersity scaling regime is much higher, $\left(B_{35 \%} / B_{1 \%}\right)_{\text {measured }}=39.2$. This may reflect an increase (of about five times) in the number of small aggregates when the larger aggregates are broken down. The polydispersity model supports a large $B$ ratio since the increase in $B$ should go as $V_{\text {large }} / V_{\text {small }}$ or $\left(R_{\text {large }} / R_{\text {small }}\right)^{3}$. The surface fractal model can not account for this large $B$ ratio.

An important feature of the data in Figure 7 is a hump at $q \approx 0.015 \AA^{-1}$, where the scattering curve deviates from the surface-fractal-like power-law regime. This indicates that the simple model of a carbon black aggregate as a single, surface-fractal structural level is somewhat naive. In fact, a more appropriate model should include two additional structural levels at higher- $q$ (smaller sizes), the primary particle level, and a graphitic scaling regime. At lowest- $q$, the data for the $1 \%$ sample (Fig. 8) follow a surface-fractallike power-law, that is, a slope of about -3.6 . A second structural level, related to the primary particles of the aggregate, at about $200 \AA$ radius of gyration (hump at $q \approx 0.015 \AA^{-1}$ ) limits this surface-fractal-like scaling regime and is followed by a Porod's Law regime, a power-law of -4 for a smooth, sharp interface. At still higher- $q$, a regime of -2 slope pertains to the 2-D graphitic substructure of the second structural level (Figs. 7 and 8). (This regime of -2 slope is best seen in the $35 \%$ sample of Fig. 7.) In Figure 8, the unified function with three levels of structure and a background, for the $1 \%$ sample, demonstrates the appropriateness of this model eq. ( $\left.1^{\prime}\right)$. Such a model agrees with electron micrographs of carbon black aggregates, which indicate the presence of a graphitic surface layer surrounding a mostly amorphous internal structure, ${ }^{6,32}$ (Fig. 1). In the unified fit, the primary particles, (Fig. 8) limit the low- $q$, surface-fractal-like power-law. Similarly, the 2-D level limits the intermediate Porod regime. As noted above, the term limits means that the power-law for the lower- $q$ level is limited at

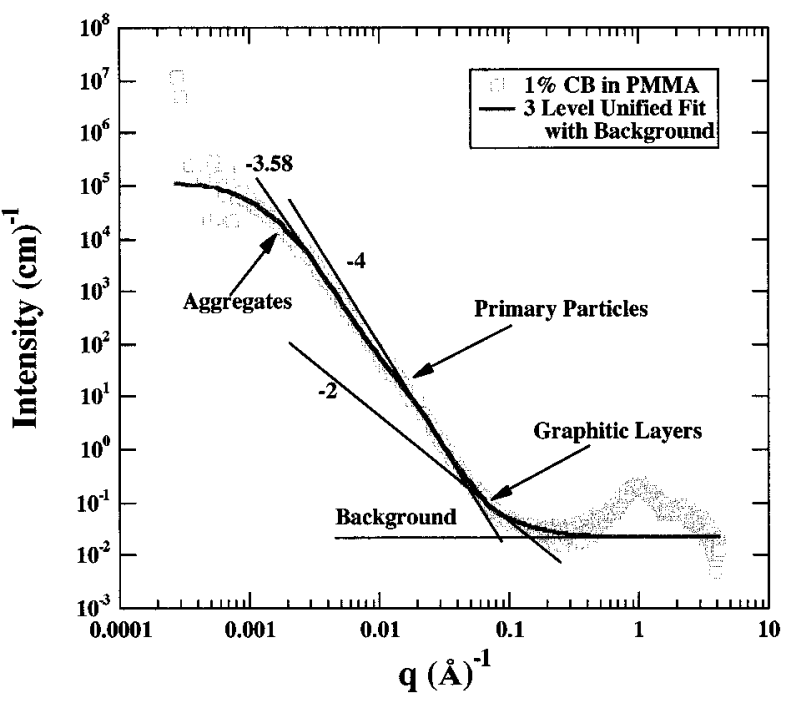

Figure 8. Unified fit to three structural levels and a background for the 1\% CB-PMMA composite.

the radius of gyration for the next level of structure following eq. ( $\left.1^{\prime}\right)$, indicating a structural relationship between the levels; that is, the larger structure is composed of the smaller structure.

The radius of gyration for the primary particles from the fit of Figure 8 is about $200 \AA$. For spherical primary particles, this yields a second moment for the diameter of about $520 \AA$. The nominal mean primary particle size for this carbon black has been reported ${ }^{36}$ to be $800 \AA$. The latter value is a fourth moment (mass average) of the diameter since mass averages are more useful in predicting carbon black performance ${ }^{6}$ Part of the discrepancy between the mean particle diameter observed in scattering and that reported for this carbon may be due to these differences in moments. A second possible source for the discrepancy pertains to the structure of the primary particles. For primary particles having a core and shell structure with an external graphitic shell covering an internal amorphous domain (Fig. 1), the observed radius of gyration might be smaller than that for a homogeneous structure since the electron density for the core and shell would be different. ${ }^{14,35}$

There are at least two interpretations of the three-level fit of Figure 8, as discussed above and as follows: a surface-fractal interpretation and a polydisperse aggregate interpretation. For the surface-fractal model, the fractally rough interface of the aggregates would be composed of smooth domains, the radius of gyration of which is close to $200 \AA$. The primary particles composing 
the surface are finally composed of graphitic layers. The polydisperse-aggregate model implies that closely packed primary particles generate smooth aggregates that range in size from close to the primary particle size to a decade larger. The distribution function for these domains would follow a power-law size distribution [eq. (6)]. If it is assumed that the observed radius of gyration for the primary particles is accurate, eq. (8) can be used to calculate the heterogeneity index for the aggregates using $P$, the largest aggregate radius of gyration, subscript 3 , and the primary particle radius of gyration, subscript 2 , in Figure 8 . The ratio of the radii of gyration can be substituted for the particle radii since these differ by only a prefactor for spherical particles. Such a calculation, for the 1\% PMMA sample, yields $H I_{\text {aggregate }}$ $=1.74$ (Table I), which is slightly low but close to literature values for similar carbon blacks, of 2.0, before mixing with the polymer matrix. ${ }^{6}$ Calculation of the heterogeneity index lends support to the polydisperse aggregate model. The heterogeneity index has also been calculated for the $5 \% \mathrm{PE}$ samples with similar results (Table I).

The unified fit parameters for the least-squares fit of Figure 8: are background $=0.0211 \mathrm{~cm}^{-1} ; G_{1}$ $=0.936 \mathrm{~cm}^{-1}, R_{g 1}=47.3 \AA, B_{1}=0.000286, P_{1}=2$, $G_{2}=137 \mathrm{~cm}^{-1}, R_{g 2}=199 \AA, B_{2}=1.45 \times 10^{-6}$, $P_{2}=4, G_{3}=1.15 \times 10^{5} \mathrm{~cm}^{-1}, R_{g 3}=0.1603 \mu \mathrm{m}$, $B_{3}=4.64 \times 10^{-6}$, and $P_{3}=3.58$. Subscript 1 refers to the 2-D graphitic layers of the primary particles, subscript 2 refers to the primary particles (or surface substructure for the surface-fractal model), and subscript 3 refers to the aggregates of $\mathrm{CB}$, which are either polydisperse or rough, as described by $P_{3}$. This three-level fit represents the minimum number of levels reasonably required to fit the entire data set. The threelevel fit was performed by an iterative process selecting well-defined regions of the scattering curve, such as the Porod or surface-fractal-like power-law regime and performing a least-squares minimization on only the parameters directly effecting these regions, holding the other fit parameters constant. This is best carried out starting with the smallest level of structure and working to the largest. A reasonable fit was obtained by visual inspection of the fit and by the absence of change in the parameters when repeating the iteration locally for each region of the curve. Due to the nature of this fitting procedure, an accurate measure of the error for each of the terms is not possible. The local fits yield very low statistical error, less than $1 \%$. The real error is probably closer to the range of values that can be obtained for the parameters. This is fairly wide for features that strongly overlap with other components; for example, the primary particle radius of gyration can vary from about 150 to about $250 \AA$ with a local least-squares fit given reasonable fixed parameters for the other components of the fit. The reported value for the primary particle radius of gyration is $200 \AA$. The fits to the low- $q$ aggregate and agglomerate levels, in the other figures of this article, are done with a single least-squares fit so that an estimate of the statistical error propagated from the data, $\sqrt{\text { counts, }}$ can be obtained, as reported in Table I. Even in Figure 8, the fit parameters for level 3 , lowest $q$, vary to a much smaller extent since level 3, the surface-fractallike regime, does not significantly overlap with other structural features.

In the diffraction regime, Figure 8 for $q>0.5$ $\AA^{-1}$, the peaks for the $35 \% \mathrm{CB}$ sample, at $q$ $\approx 1.77,2.43$, and $2.88 \AA^{-1}$, and broader CB peak at $2.07 \AA^{-1}$ in Figures 7 and 8 are related to the ordered graphitic and amorphous components of $\mathrm{CB}$, respectively. The dependence of these peaks on CB loading (Fig. 7) can be used to identify these peaks with the CB component. The broad hump at about $0.95 \AA^{-1}$ is the amorphous halo of PMMA.

The existence of a Porod regime, power-law of -4 slope, for the primary particle level, subscript 2 in the fit to Figure 8, also can be verified from SANS on the CB-PE composite. For SANS, the contrast for $\mathrm{PE}$ is essentially zero such that the superposition of the lamellar correlations of $\mathrm{PE}$ are removed from the data. ${ }^{37}$ For this case, it is expected that only the CB domains will be observed. Similar morphological features to those observed in Figure 8 for the PMMA-CB composite are observed in Figure 9 for the CB-PE composite. The main features in this regime are the smooth, sharp interfaces of the primary particles.

\section{5\% Carbon Black-PE Composites}

The $35 \%$ carbon black-PE composites (Fig. 10) show significant differences in scattering from the 5\% samples (Figs. 4-6), and the 1\% PMMA sample (Figs. 7 and 8). The Guinier knee for the aggregate level has shifted to higher $q$ in the $35 \%$ $\mathrm{PE}$ composites, indicating a reduction in size for the largest aggregates, $q \approx 2 \pi / D$. Additionally, there appears to be a fairly broad low-dimensional scaling regime at lowest $q$, reminiscent of 


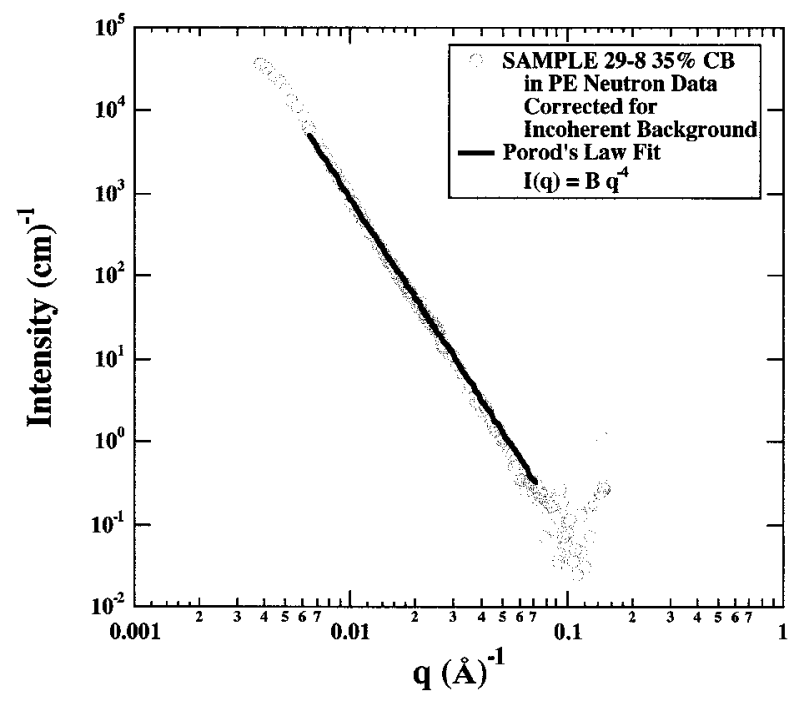

Figure 9. Small-angle neutron scattering data, which verify the Porod regime of Figure 6. The Porod prefactor is $B$ equals $8.56 \times 10^{-6} \AA^{-4} \mathrm{~cm}^{-1}$ in these data. The $q$-range for SANS is much narrower than in the other figures, which combine Bonse-Hart, SAXS, and X-ray diffraction.

the 35\% PMMA sample (Fig. 7). As in the 35\% PMMA sample, a model was used based on large aggregate break up, on milling the higher-concentration samples, followed by agglomeration of these partially decomposed aggregates into fairly linear agglomerates (Fig. 1). A low- $q$ fit for the $35 \%$ carbon black-PE composite at $200^{\circ} \mathrm{C}$ (Fig. 10) uses a two-level model: low- $q$, linear agglomerates with higher- $q$ power-law polydisperse aggregates (Table I). A similar fit has been performed for the room-temperature 35\% PE composite. The thermal dependence seen in scattering is completely reversible with the initial room temperature data overlapping exactly with the cooled data shown in Figure 10.

The Guinier prefactor ratio, $G_{200{ }^{\circ} \mathrm{C}, 35 \%} / G_{R T, 35 \%}$, for the aggregates in Figure 10 is 1.78, indicating a local enhancement of concentration from $35 \%$ in the melt (assuming a random distribution) to about $80 \%$ in the crystalline matrix for the largest aggregates, using eq. (11). A similar enhancement is seen for the surface-fractal-like power-law prefactor $(81 \%)$ and for the low- $q$, agglomerate scaling regime (79\%) (Table I). For true surface fractals, the increase in $B$ should be about $26 \%$ (Guinier and Fournet, ${ }^{14}$ page 80 ), accounting only for the change in matrix contrast on melting. The aggregate power-law regime displays a slope close to -4 , which may be associated with surface- dominated aggregate decomposition mechanism, which is expected to lead to $N(r)=C r^{-2}$, and $P$ $=4$, as discussed above. The aggregate powerlaw of -4 was fixed in the fits [Table I (free fits yield a slope close to -4 )]. It should be noted that the fitting range between the primary particle and largest aggregate radii of gyration is narrower than in the fits to the $5 \%$ samples, which may contribute to error in this slope. The enhanced $B$ ratio for the $35 \%$ samples on melting of the matrix cannot be explained using the surfacefractal model. These values for the $G$ and $B$ ratios on melting the matrix are described well by the polydisperse aggregate model.

\section{CONCLUSIONS}

Scattering data from composite materials are useful for investigating structural models in complex systems where microscopy is limited by overlap of structural features in three dimensions, multiple levels of structure, absorption for optical microscopy, and conductivity/charge build up issues for electron microscopy. Moreover, scattering offers access to scaling features, such as a description of certain types of polydispersity and fractal scaling, if the correct model for the composite system can be determined. This article addresses several issues in carbon black-polymer composites. First,

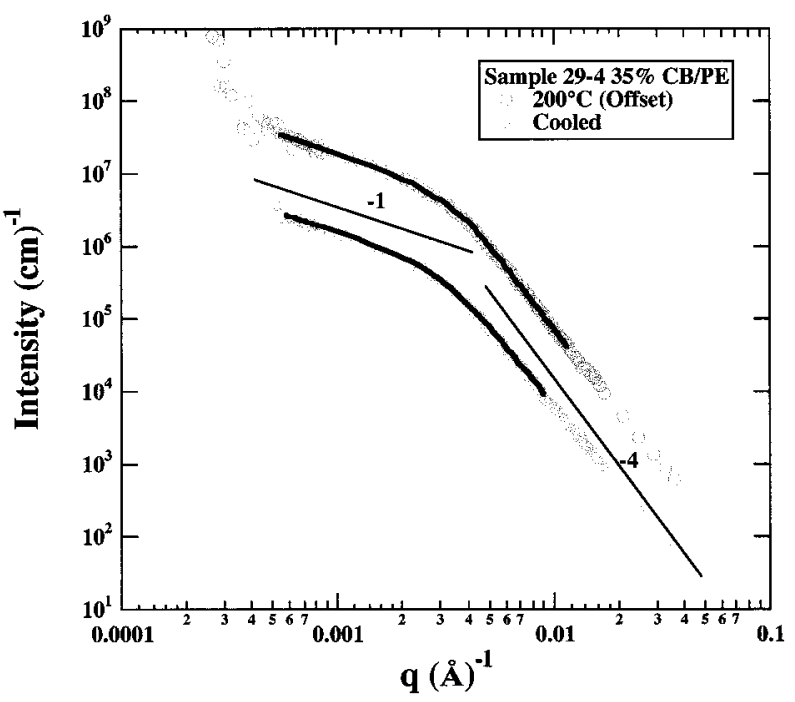

Figure 10. $35 \%$ CB-PE composite at $200^{\circ} \mathrm{C}$ and cooled to room temperature. The fit is to equation $\left(1^{\prime}\right)$ with two levels of structure. $200^{\circ} \mathrm{C}$ data have been offset (multiplied by 8) but would be higher in intensity than the cooled run, even if not offset. 
nanoscale features observed in SAXS data from carbon black-polyethylene composites are probably due to semicrystalline features in the matrix polymer (Figs. 4 and 5). Second, it was shown that power-law polydispersity in aggregate size can account for the observed surface-fractal-like aggregate scaling regime [Fig. 2 and eq. (7)]. This model is supported by a high polydispersity in aggregate size from microscopy and by the lack of convincing evidence for surface-fractal features in micrographs. Moreover, it is possible to calculate a reasonable heterogeneity index for the aggregates using a power-law polydispersity model [eq. (8) and Table I]. Third, scattering from carbon black in an amorphous polymer matrix was used to estimate the primary particle size and other nanosized structural features of the carbon black, as well as to support a model based on changes in large scale agglomeration, and on aggregate break up at higher concentrations (Figs. 7 and 8). If X-ray diffraction data are combined with SAXS data, it is possible to identify a scaling regime associated with graphitic layers in the primary particles. Fourth, differences in scattering between low and high concentrations of carbon black in PE support a model based on large aggregate break up and an associated linear agglomeration of aggregates in high-concentration carbon black samples. The latter may be critical to understanding conductivity switching in these composites. Finally, the observed changes in scattering contrast between the melt and semicrystalline PE matrix can be modeled in terms of a significant enhancement in local concentration of carbon black aggregates, associated with linear agglomerates, as shown in Figure 1 and modeled using eq. (11). Linear agglomerates may be enhanced in the presence of the expected linear crystalline fiber bundles of $\mathrm{PE}$ (Fig. 1).

G. Beaucage was supported by a PRF-G grant from the Petroleum Research Fund of the American Chemical Society. This work relied heavily on national user facilities at the National Institute of Standards and Technology, Oak Ridge National Laboratories, and at Brookhaven National Laboratories (NIST beam-line X23-A3 for Bonse-Hart data). Discussions with Dr. Charles R. Herd of Columbian Chemicals Corporation proved to be extremely valuable in the interpretation of these data, and he is acknowledged for his contribution to this article. The contribution of G. D. Wignall in his assistance with collection of SANS data and in discussions concerning this article are greatly appreciated and acknowledged. K. Schwartz and M. Wartenberg of
Raychem Corporation supplied the samples used in this article and participated in extensive discussions concerning this article, as well as providing micrographs of the carbon and supporting information. They are acknowledged for their significant effort in contributing to this article.

\section{REFERENCES AND NOTES}

1. Probst, N. Eur Rubber J 1984, 11, 4.

2. Medalia, A. I. in Carbon Black-Polymer Composites, Sichel, E. K., Ed.; Marcel Dekker: New York, 1982; p 1.

3. Probst, N. in Carbon Black, 2nd Ed.; Donnet, J.-B.; Bansal, R. C.; Wang, M.-J., Eds.; Marcel Dekker: New York, 1993; p 271.

4. Wignall, G. D.; Farrar, N. R.; Morris, S. J Mater Sci 1990, 69, 75 .

5. Marr, D. W. M.; Wartenberg, M.; Schwartz, K. B.; Agamalian, M. M.; Wignall, G. D. Macromolecules 1997, 30, 2120.

6. Herd, C. R.; Hess, W. M. in Carbon Black, 2nd ed.; Donnet, J.-B.; Bansal, R. C.; Wang, M.-J., Eds.; Marcel Dekker: New York, 1993; pp 89-173.

7. Long, G. G.; Jemain, J. R.; Weertman, J. R.; Black, D. R.; Burdette, H. E.; Spal, R. J Appl Cryst 1991, 24, 30-39.

8. Rieker, T.; Hurd, A.; Schaefer, D. W.; Hua, D.-W.; Beaucage, G. to appear.

9. Russell, T. P.; Lin, J. S.; Spooner, S.; Wignall, G. D. J Appl Cryst 1988, 21, 629.

10. Wignall, G. D.; Agamalian, M. Presented at the American Crystallographic Association Meeting, Saint Louis, MO, 7/1997.

11. Beaucage, G.; Schaefer, D. W. J Non-Cryst Solids 1994, 172-174, 797.

12. Beaucage, G. J Appl Cryst 1995, 28, 717-728.

13. Beaucage, G. J Appl Cryst 1996, 29, 134.

14. Guinier, A.; Fournet, G. Small-Angle Scattering of X-rays; John Wiley and Sons: New York, 1955; p 27.

15. Bale, H. D.; Schmidt, P. W. Phys Rev Lett 1984, 53, 596-599.

16. Wong, P.; Bray, A. J. J Appl Cryst 1988, 21, 786794.

17. Schmidt, P. W. J Appl Cryst 1992, 15, 567-569.

18. Hurd, A. J.; Schaefer, D. W.; Smith, D. M.; Ross, S. B.; Le Mehaute, A.; Spooner, S. Phys Rev B 1989, 39, 9742-9745.

19. Bansal, R. C.; Donnet, J.-B. Carbon Black, 2nd Ed.; Donnet, J.-B.; Bansal, R. C.; Wang, M.-J., Eds.; Marcel Dekker: New York, 1993; pp 67-88.

20. Donnet, J.-B.; Voet, A. Carbon Black; Marcel Dekker: New York, 1976.

21. Lahaye, J.; Prado, G. Chemistry and Physics of Carbon, Vol. 14; Walker, P. L., Jr., Ed.; Marcel Dekker: New York; 1978, p 168. 
22. Lahaye, J. Polym Degrad Stab 1990, 30, 111.

23. Gordon, A. S.; Smith, S. R.; McNesby, J. R. 7th Symposium (International) on Combustion; Oxford Press: London, 1959, p 317.

24. Ball, R. J.; Howard, J. B. 13th Symposium (International) on Combustion; The Combustion Institute: Pittsburgh, PA, 1971, p 353.

25. Wersborg, B. L.; Howard, J. B. "14th Symposium (International) on Combustion"; The Combustion Institute: Pittsburgh, PA, 1973, p 929.

26. Glassman, I.; Yaccarino, P. Combust Sci Technol 1980, 24, 107.

27. Santoro, R. J.; Semerjian, H. C.; Dubbins, R. A. Combust Flame 1983, 51, 203.

28. Harris, S. J.; Weiner, A. M. Combust Sci Technol 1983, 32, 15.

29. Harris, S. J.; Weiner, A. M. Combust Sci Technol 1983, 32, 26.

30. Harris, S. J.; Weiner, A. M. Combust Sci Technol 1984, 38, 75 .
31. ASTM D 3849. ASTM Annual Book of Standards, Vol. 09.01; ASTM Institute for Standards Research, ASTM: West Conshohocken, PA, 1990; p 630.

32. Heidenreign, R. D.; Hess, W. M.; Ban, L. L. J Appl Cryst 1968, 1, 1.

33. Beaucage, G.; Aubert, J. H.; Lagasse, R. R.; Schaefer, D. W.; Rieker, T. P.; Ehrlich, P.; Stein, R. S.; Kulkarnni, S.; Whaley, P. J Polym Sci, Part B: Polym Phys 1996, 34, 3063-3072.

34. ASTM D-2414-90. ASTM Annual Book of Standards, Vol 09.01; The ASTM Institute for Standards Research, ASTM: West Conshohocken, PA, 1990; p 434.

35. Feigin, L. A.; Svergun, D. I. Structure Analysis by Small-Angle X-ray and Neutron Scattering; Plenum Press: New York, 1987.

36. Herd, C. R. Columbian Chemicals Company, Schwartz, LA. personnel communication, 1996.

37. Wignall, G. D. in Physical Properties of Polymers Handbook; Mark, J. E., Ed., American Institute of Physics Press: Woodbury, NY, 1996; p 299. 